\title{
On radio frequency current drive in the ion cyclotron range of frequencies in DEMO and large ignited plasmas
}

\author{
Marco Brambilla, Roberto Bilato \\ Max-Planck Institut für Plasmaphysik, D-85748 Garching, Germany \\ E-mail: roberto (dot)bilato (at) ipp(dot)mpg(dot)de
}

\begin{abstract}
To explore the possibility of efficient Fast Wave current drive in an ignited plasma in the Ion Cyclotron (IC) range of frequency in spite of competition from absorption by ions, we have added to the full-wave toroidal code TORIC a set of subroutines which evaluate absorption by these particles at Ion Cyclotron harmonic resonances, using a realistic "slowing-down" distribution function, and taking into account that their Larmor radius is comparable or even larger than the Fast Wave wavelength.

The thermalized population of $\alpha$-particles is not a serious competitor for power absorption as long as their number density is compatible with maintenance of ignition. By contrast, the energetic slowing down fraction, in spite of its even greater dilution, can absorb from the waves a substantial amount of power at the cyclotron resonance and its harmonics. An extensive exploration both in frequency and in toroidal wavenumbers using the parameters of one of the European versions of DEMO shows that three frequency windows exist in which damping is nevertheless predominantly on the electrons. Designing an antenna capable of shaping the launched spectrum to optimize current drive, however, will not be straightforward. Only in a narrow range when the first IC harmonic of Tritium is deep inside the plasma on the highfield side of the magnetic axis, and that of Deuterium and Helium is still outside on the low-field side, it appears possible to achieve a satisfactory current drive efficiency with a conventional multi-strap antenna, preferentially located in the upper part of the vessel. Exploiting the other two windows at quite low and quite high frequencies is either impossible on first principles, or will demand novel ideas in antenna design.
\end{abstract}

PACS numbers: $52.25 \mathrm{Fi}, 52.25 \mathrm{Ya}, 52.55 \mathrm{Fa}$

Keywords: ICRF, fast-wave, current drive, fusion reactor, high-harmonics, alphaparticles 


\section{Introduction}

To maintain an ignited tokamak plasma in steady-state, or at least to prolong as much as possible the pulse duration, in addition to the boostrap current, some non-ohmic current drive (CD) is regarded as necessary [1]. The CD method must be compatible with the environment of the ignited plasma, and its efficiency must be sufficiently high not to compromise the global power balance of the reactor. In the present planning phase of the DEMO reactor [2], it is therefore natural to explore the advantages and limitations of the various options available for non-ohmic CD, taking into account these requirements. In the Ion Cyclotron (IC) range of frequencies radio frequency ( $\mathrm{RF}$ ) power is readily available, and can be used also for auxiliary ion heating during start-up. Competition from absorption by ions, on the other hand, in particular by thermonuclear alpha $(\alpha)$ particles, is undesired for CD once ignition is reached. Indeed, IC heating of $\alpha$-particles, even on a scale which would by itself not spoil the global power balance, might put a non negligible number of these particles on deeply trapped orbits, leading to rapid losses of energetic particles and possible damage to the wall.

To investigate the question with a model as realistic as possible, two extensions of the TORIC -SSFPQL package [3, 4] have been made. First, we have added a subroutine which evaluates the distribution function of the thermonuclear produced $\alpha$-particles using the kinetic model summarized in the next section. Secondly, and more importantly, the model of Fast Wave (FW) damping at high IC harmonic resonances implemented in TORIC [5] has been extended to include the effects of the diluted but very energetic population of slowing-down $\alpha$-particles. This has required to modify the model so that arbitrary distribution functions can be taken into account, and IC absorption can be evaluated to all orders in the Larmor radius also for the fundamental and the first IC harmonic. This extension is presented in section 3. It is also worth mentioning that in TORIC the solution of Maxwell's equations for many different toroidal wavenumbers $n_{\varphi}$ can now be performed in parallel with the MPI protocol. The results of such a scan can then be superposed with weights depending on the antenna geometry. This is useful for a study like the present, since it allows to investigate the constraints imposed by the physics on the antenna design.

The current drive efficiency has been estimated using the well-known Ehst-Karney (E-K) parametrization [6], which takes into account the effects of the wave polarization and of toroidal trapping of resonant electrons. A systematic comparison of this parametrization with the results of a kinetic code in toroidal geometry performed by Wright et al. [7] has confirmed its wide applicability in spite of a certain number of simplifications. In particular, the E-K parametrization does not take into account exactly the details of the magnetic configuration, such as the ellipticity of the crosssection. Nevertheless, these inaccuracies are expected to be of second order in the inverse aspect ratio. The E-K parametrization has been compared with the results of a Fokker-Planck solver for ASDEX Upgrade [8], whose cross section has an ellipticity comparable to DEMO, and found in very good agreement. We have not used the 

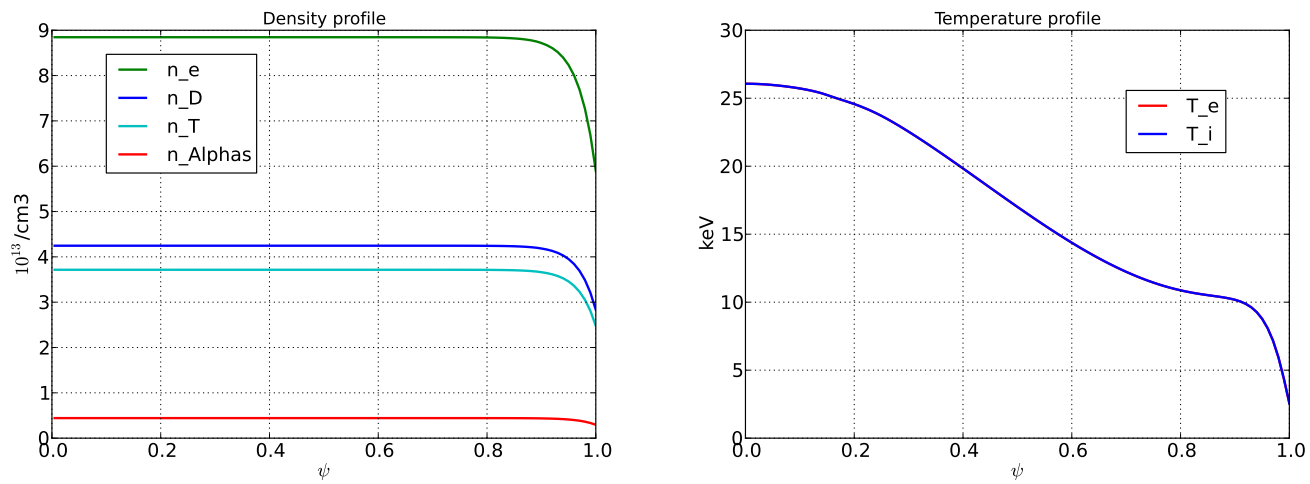

Figure 1. Electron and ion density and temperature profiles (all temperatures are equal) of the reference DEMO discharge. In the abscissa $\psi-r / a$ along the outer equatorial plane.

Fokker-Planck solver of [8] in the present study, because is not automatically coupled with TORIC, and would have greatly increased the already large computational load.

As just mentioned, the main difficulty for FWCD in this frequency range is competition by ion absorption at IC harmonics. The conclusions of our exploration is that while frequency windows in which damping is predominantly on the electrons do exist, they are narrow and inconveniently located in frequency, in the sense that it will not be easy to design an antenna capable of selectively launching waves with parallel phase velocity appropriate to optimize the driven current. Before presenting the results of the simulations in section 4, these conclusions can be substantiated here by elementary considerations. For concreteness, we base this discussion on the DEMO parameters, but the conclusions hold for any large ignited plasma.

The DEMO version which we have used for our simulations has major radius $R=9 \mathrm{~m}$, minor horizontal radius $a=2.25 \mathrm{~m}$, ellipticity 1.73 , and central magnetic field $B$ of 6.8 Tesla. Density and temperature profiles are shown in figure (1). With these parameters, the first IC resonance (the fundamental of Tritium) enters the plasma at $f \simeq 25 \mathrm{MHz}$ (actually, about $1 \mathrm{MHz}$ earlier because of the Doppler width of the absorption region). Hence, to guarantee pure electron damping the frequency must be lower than $24 \mathrm{MHz}$. Above this frequency absorption by ions is mostly dominant; the frequency scans presented in section 4, nevertheless, suggest that it might be possible to minimize competition by ions in two other frequency windows. The first is a narrow range around 75-80 MHz, depending on the poloidal location of the launcher. In this window the first harmonic resonance of Tritium is near the inner plasma edge, well screened from the antenna by the intervening electron damping, while the first IC harmonic of Helium and Deuterium is still outside the plasma on the low field side. With rather severe demands on the antenna selectivity, a third window is available above about 180-200 MHz. In all three ranges, however, rather stringent conditions have to be satisfied by the parallel power spectrum radiated by the antenna.

The well-known curves of the CD efficiency presented by Ehst and Karney [6] show 
that for successful CD the normalized phase velocity $x_{e}=\omega / k_{\|} v_{t h e}$ of the waves should be as large as possible, and in any case should not descend much below unity, in order to avoid degradation of the CD efficiency by toroidal trapping of resonant electrons (for consistency with TORIC, we use here the definition $v_{\text {the }}=\left(2 T_{e} / m_{e}\right)^{1 / 2}$, so that $x_{e}$ is $\sqrt{2}$ times the variable $w$ in the Ehst and Karney paper). This practically rules out the possibility of efficient current drive in the lowest frequency window below about $25 \mathrm{MHz}$. Indeed, the condition $x_{e} \gtrsim 1$, expressed in terms of toroidal wavenumbers, is equivalent to

$$
n_{\varphi} \lesssim \frac{f_{[\mathrm{MHz}]} R_{[\mathrm{m}]}}{3 \sqrt{T_{e[\mathrm{keV}]}}}
$$

With the reference parameters of DEMO, this means that at $20 \mathrm{MHz}$ the antenna toroidal power spectrum should be peaked around $n_{\varphi} \lesssim 12$. An antenna capable of imposing such a periodicity would be far too wide for the available access.

The opposite difficulty is encountered in the highest part of the frequency range covered by our exploration. As will be seen in section 4, in this range damping by energetic $\alpha$-particles at the fourth and fifth IC harmonic is far from negligible unless the antenna selects toroidal wavenumbers such that $x_{e} \lesssim 0.5$, which at $200 \mathrm{MHz}$ means $n_{\varphi} \gtrsim 130$. In this case reduction of the CD efficiency by toroidal effects, although not negligible, is not as severe as in the low-frequency range, but the required periodicity is hardly to be obtained with conventional multi-straps antennas. Novel antenna designs will have to be developed to take advantage of this frequency window.

It is worth mentioning that the latter windows extends to appreciably higher frequencies, outside the reach of TORIC; a study of this range with ray tracing has recently been performed by Prater et al. [9], neglecting ion absorption altogether, and using the Ehst-Karney parametrization (checked with a Fokker-Planck solver in a few cases) to estimate CD. As pointed out in this work, at these frequencies a multi-element combline antenna might be a valid choice.

Constraints on the antenna geometry are less severe in the intermediate frequency window. It is also interesting that in this range CD is predicted to be more efficient if the antenna is located in the upper part of the vessel rather than on the equatorial plane [10]. This window, however, is rather narrow, and its exact position in frequency depends sensitively on the value of the static magnetic field. The rf system, therefore, will have to be rather flexible to cope with possible variations of the equilibrium parameters. On the other hand, only in this window it is conceivable to use the same system to heat ions during start-up, and to drive current after ignition.

The three windows where FWCD is in principle possible have been already identified by Hannan et al. [11] and Lerche et al. [10]. In these works FW absorption by the energetic $\alpha$-population is evaluated for simplicity assuming that their distribution function can be modeled by an equivalent Maxwellian having the same number density and the same energy content as the distribution function of the slowing-down population. This option has been retained also in TORIC : in section 4.4 we will show that the results obtained with the equivalent Maxwellian reproduce qualitatively the trends predicted 
using the more realistic slowing-down distribution function, but rather large quantitative differences are observed in the power repartition between the various species and in the amount of current driven.

It must be mentioned, however, that also the present investigation is incomplete in this respect, because the distribution function of energetic $\alpha$-particles used to estimate IC absorption takes into account only collisions with the background plasma, but not the effects of IC heating itself. The SSFPQL Fokker-Planck-Quasilinear solver coupled with TORIC $[3,4]$ which iteratively evaluates the selfconsistent ion distribution function in the presence of IC heating admits only fundamental and first harmonic resonances. The surface-averaged model implemented in SSFPQL, moreover, is inadequate to deal with IC heating of particles with energies in the MeV range: a more sophisticated method would be required, such as the Monte Carlo approach applied by Choi et al. [12] to the simulation of DIII-D experiments. While for thermal ions the importance of deviations from Maxwellians are likely to decrease rapidly with increasing harmonic order, this is not necessarily true at the very large energies of slowing down $\alpha$-particles. It is to be expected, therefore, that quasilinear effects on slowing-down $\alpha$-particles will make efficient current drive even more difficult than presently predicted. Nevertheless, just in the frequency windows identified for CD the power per particle deposited in the energetic $\alpha$-particles is so low that quasilinear effects are unlikely to change much our conclusions.

The rest of this paper is organized as follows. In section 2 we introduce the distribution function of themonuclearly produced $\alpha$-particles, and in section 3 we discuss how absorption by these particles at IC resonances is modeled in TORIC. The main results relevant for current drive in the DEMO ignited plasma are presented in section 4 , based on a large set of simulations of wave propagation and absorption in the frequency range from 20 to $300 \mathrm{MHz}$. Some conclusions and considerations for further work are presented in the final section.

\section{The distribution function of thermonuclear $\alpha$-particles}

Since absorption of RF waves by $\alpha$-particles depends critically on their distribution function, and, in particular, on the concentration of its high energy component, it is necessary to determine $f_{\alpha}(v, \psi)$ in a sufficiently reliable way as function of both energy and radial position throughout the entire plasma. As discussed in [13], there is a sharp distinction between a bulk part in thermal equilibrium with the background ion species, known as the ashes, and a highly energetic population, slowing down mainly on the electrons. The concentration of the latter is proportional to the thermonuclear birth rate, while above a modest concentration the radial density profile of the ashes is proportional to the density profile of all other ions. For energies larger than a few times the temperature of the plasma, moreover, the distribution function of the suprathermal population is predicted to be proportional to $\left(v^{3}+v_{\text {crit }}^{3}\right)^{-1}$ up to the birth energy $[14,15]$.

Adapting the model proposed by Stix to describe the slowing-down of thermonuclear 
alpha particles [16], the Fokker-Planck equation can be integrated analytically [17]

$$
f_{\alpha}(v)=e^{-v^{2}}\left[\frac{n_{\mathrm{ash}}}{\pi^{3 / 2}}+\frac{S_{\mathrm{tn}}}{2 \pi \nu_{\mathrm{ref}}} \int_{0}^{v} \frac{e^{u^{2}}}{u \Psi(u)}\left(H\left(u_{\mathrm{b}}-u\right)-(1-\Phi(u))\right) \mathrm{d} u\right]
$$

where the velocity is normalized to the thermal velocity $v_{t h \alpha}=\left(2 T / m_{\alpha}\right)^{1 / 2}$ of the thermal $\alpha$ s (we have assumed for simplicity all charged species to have the same temperature, but the general case requires only a simple modification of the argument of the two exponentials). Here

$$
\Psi(v)=\sum_{\beta} \frac{\nu_{\alpha \beta}}{\nu_{\mathrm{ref}}} \frac{\Phi\left(\gamma_{\alpha \beta} v\right)}{\gamma_{\alpha \beta}^{2} v^{2}}
$$

with $\gamma_{a \beta}=v_{t h \alpha} / v_{t h \beta}$ and

$$
\Phi\left(u_{\beta}\right)=\frac{4}{\sqrt{\pi}} \int_{0}^{u_{\beta}} u^{2} e^{-u^{2}} d u=-\frac{2}{\sqrt{\pi}} u_{\beta} e^{-u_{\beta}^{2}}+\operatorname{Erf}\left(u_{\beta}\right)
$$

The summation in eqn (2) is over all other ion species (including ${ }^{4} \mathrm{He}_{++}$ashes) and electrons. In these expressions

$$
\nu_{\alpha \beta}=\frac{4 \pi Z_{\alpha}^{2} Z_{\beta}^{2} e^{4} n_{\beta} \Lambda^{\alpha \beta}}{m_{\alpha}^{2} v_{t h \alpha}^{3}} \quad \nu_{\mathrm{ref}}=\sum_{\beta} \nu^{\alpha / \beta}
$$

The Heaviside function $H\left(u_{\mathrm{b}}-u\right)$ in eqn (1) is due to the source term

$$
S_{\mathrm{tn}}=n_{D} n_{T}\langle\sigma v\rangle \delta\left(v-v_{\mathrm{b}}\right)
$$

describing the birth rate of the $\alpha \mathrm{s}$, where $v_{\mathrm{b}}=\left(2 E_{\alpha} / m_{\alpha}\right)^{1 / 2}$, with $E_{a}=3.52 \mathrm{MeV}$, is the $\alpha$-particles birth velocity. To have a steady-state solution, finally, a term $S_{\text {loss }}(v)$

$$
S_{\mathrm{loss}}(v)=-\frac{S_{\mathrm{tn}}}{\nu_{\mathrm{ref}}} f_{M \alpha}(v)=-\frac{S_{\mathrm{tn}}}{\nu_{\mathrm{ref}}} \frac{e^{-v^{2}}}{\pi^{3 / 2}}
$$

has been included, describing losses of thermalized $\alpha$-particles, designed to compensate their production by fusion in steady-state. This loss term generalizes slightly that used by Stix [16], namely a sink localized at zero velocity.

The distribution function (1) is plotted in fig. 2 (this figure has actually been made by specializing to the case isotropic in velocity the Fokker-Planck solver SSFPQL coupled to TORIC $[18,3]$; this explains the presence of a few $\alpha$-particles beyond the birth energy). This figure clearly shows the splitting of $f_{a}$ into ash and slowing-down part discussed by [13]. The forced term in eqn (1), proportional to the birth rate $S_{\mathrm{tn}}$, represents the fast population driven by their creation at $3.5 \mathrm{MeV}$; because of the sink, this term vanishes at $v=0$. The ash density $n_{\text {ash }}(\psi)$ is a constant of integration which can depend in an arbitrary way on the radial position. Following Angioni et al. [13], it was taken proportional to the density of the other ions. In all the results presented in the following the ashes have been assumed to be $5 \%$ in number. The radial density 


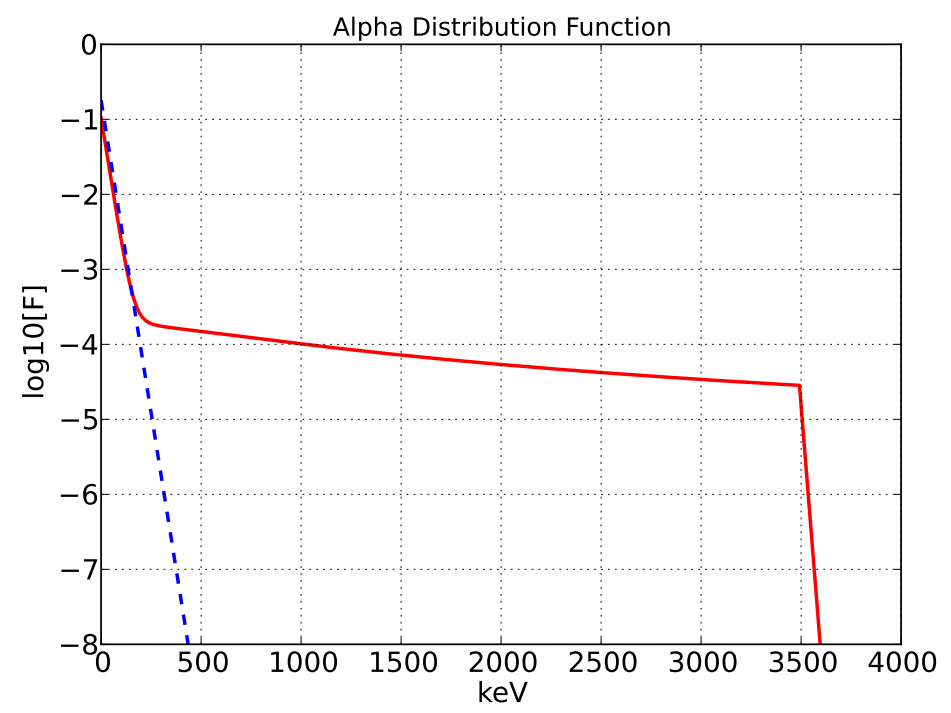

Figure 2. Distribution function (normalized to unity) of the $\alpha$-particles predicted by eqn (1) in the core of the reference DEMO discharge. The concentration of ${ }^{4} \mathrm{He}$ ashes has been assumed to be $5 \%$ in number.

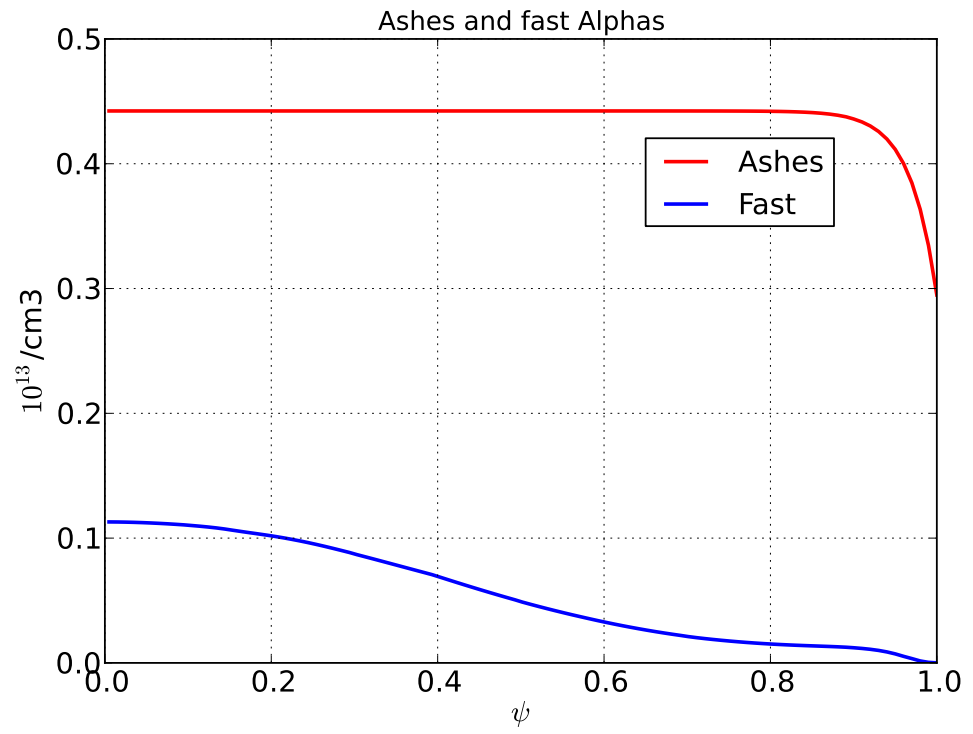

Figure 3. Radial profiles of ${ }^{4} \mathrm{He}$ ashes, and of fast $\alpha$-particles predicted by eqn (8) for the reference DEMO scenario. The ashes concentration is assumed to be $5 \%$. 
profiles of slowing down and thermalized ${ }^{4}$ He ions obtained from eqn (1) are shown in fig. 3. Scans at $2 \%$ ashes concentration give very similar results; at $10 \%$ reduction of the energetic $\alpha$-population due to dilution of the $\mathrm{D}$ - $\mathrm{T}$ fuel begins to be important.

The sharp distinction between energetic $\alpha$-particles and ashes in fig. 2 suggests to separate completely the two populations. At energies sufficiently above thermal one can neglect collisional diffusion compared to friction; in the range $v_{t h i} \ll v \ll v_{\text {the }}$, moreover, $\gamma_{\alpha i} u \gg 1$ and $\gamma_{\alpha e} u \ll 1$. Exploiting these inequalities, the forced term of eqn (1) can be approximated as [14]

$$
f_{\mathrm{tn}}(v) \simeq \frac{S_{\mathrm{tn}}}{\nu_{\alpha e} B_{\tau}}\left\{\begin{array}{ll}
\frac{\Phi(v)}{v^{3}+v_{\text {crit }}^{3}} & v \leq v_{\mathrm{b}} \\
0 & v>v_{\mathrm{b}}
\end{array} \quad v_{\text {crit }}=\left(\frac{A_{\tau}}{B_{\tau}}\right)^{1 / 3}\right.
$$

with

$$
A_{\tau}=\frac{\nu_{\alpha i}}{\nu_{\mathrm{ref}}} \frac{T_{\alpha}}{T_{i}} \frac{1}{\gamma_{\alpha i}^{2} v^{2}} \quad B_{\tau}=\frac{4}{3 \sqrt{\pi}} \frac{\nu_{\alpha e}}{\nu_{\mathrm{ref}}} \frac{T_{\alpha}}{T_{e}} \gamma_{\alpha e}^{3}
$$

(the subscript 'tn' indicates that we are considering only the forced term in eqn (1); the factor $\Phi(u)$ is usually omitted from the slowing down distribution function, but keeping it gives a better approximation in the whole range $0 \leq v \leq v_{\text {crit }}$ ).

In figures 4 and 5 the slowing down distribution function $f_{\mathrm{tn}}$ is compared to the forced part of the distribution (1). The agreement is excellent in the hot core region of the plasma, and degrades slightly only in the plasma periphery. The reason is that the energy domain of validity of the inequality $v \ll v_{\text {the }}$ justifying the approximation (8) shrinks as the plasma temperature decreases. In the simulations, the parameters of the slowing-down distribution are determined by fitting to the SSFPQL solution, rather than using the theoretical values (9).

Also shown in fig 4 is the "equivalent Maxwellian", namely a Maxwellian having the same energy content as the slowing-down distribution. To our knowledge, the equivalent Maxwellian has been used in all previous investigations of IC current drive in an ignited plasma $[19,11,10,20]$. This has the advantage that the velocity integrals in the contributions of the $\alpha$-particles to the coefficient of the wave equations can be evaluated analytically (cfr. the next section). Computationally, this reduces the execution time of TORIC by at least a factor 2. It will be seen in section 4 , however, that the results obtained in this way are only qualitatively correct. Except for comparison with the equivalent Maxwellian, in all runs of TORIC we have used the slowing-down distribution (8), together with the Maxwellian distribution for the ashes.

\section{Modeling wave damping by higher Ion Cyclotron harmonic resonances}

To investigate the competition between ions and electrons for absorption of RF waves in the IC range of frequencies, a reliable model of ion absorption at IC harmonic resonances in toroidal geometry is needed. This implies taking into account large Larmor radius 


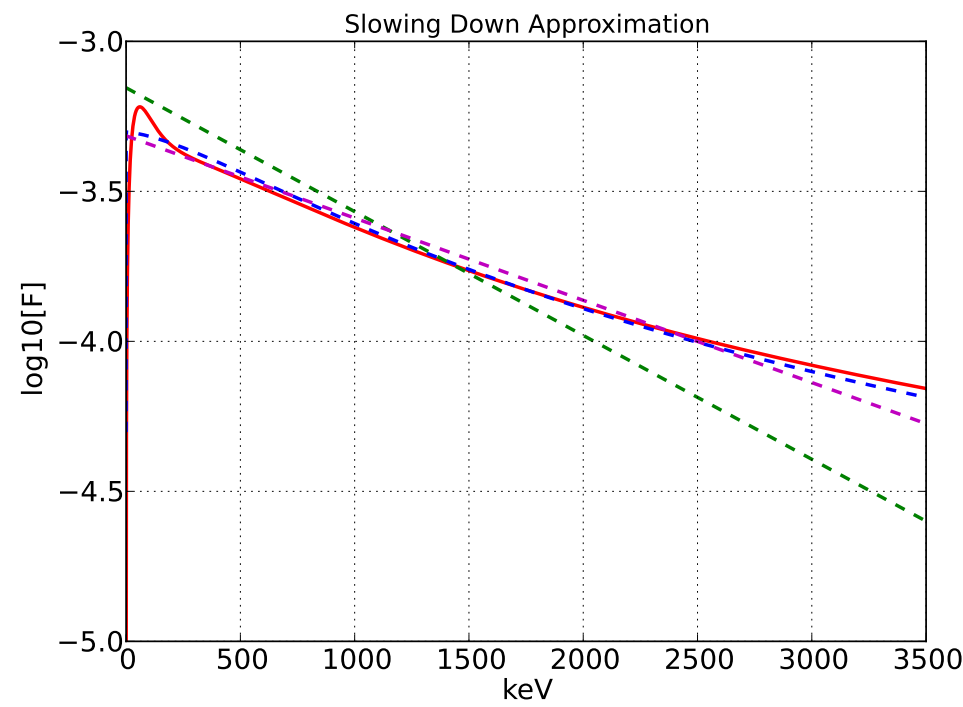

Figure 4. The forced part of eqn (1) on axis of the DEMO reference scenario (full red), and its approximation by the slow-down distribution (dashed blue), both normalized to unity. The dashed magenta line is the equivalent truncated Maxwellian, and the green dashed line the equivalent Maxwellian without truncation.

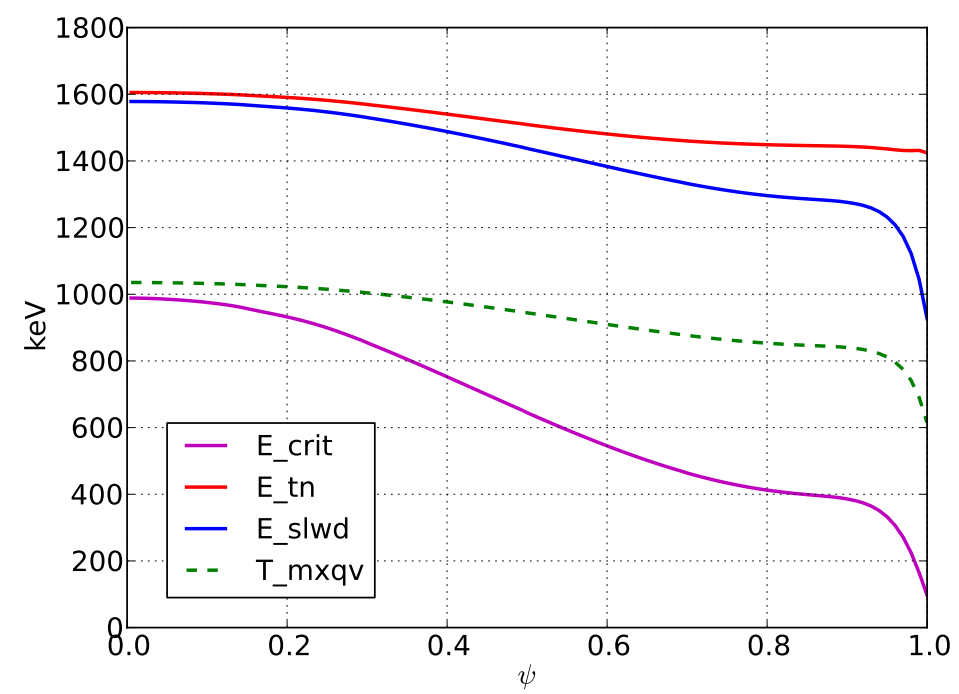

Figure 5. The radial profiles of $E_{\text {crit }}$ (full magenta), of the energy content $E_{\text {tn }}$ of the forced part of eqn (9) (full red), and of the slowing down approximation $f_{\alpha}^{\text {sd }}\left(E_{\text {sldwn }}\right.$, full blue). The dashed green curve is the temperature of the equivalent Maxwellian $\left(E_{\mathrm{tn}} \simeq 3 T_{\mathrm{meqv}} / 2\right)$. 
contributions to the wave equations. When this is the case the wave equations obtained by solving the linearized Vlasov equation are integro-differential equations. To derive an acceptable approximation which can be implemented without altering the structure of the equations solved by TORIC it is necessary to assume that resonances at harmonics higher than the second ( $\omega=p \Omega_{c i}$ with $p \geq 3$ ) affect only the $\mathrm{FW}$. This amounts to neglect local excitation of higher order Ion Bernstein (IB) waves near the corresponding resonances (these waves have wavelength so short that they could never be resolved by any reasonable mesh). With this simplification, absorption at higher IC harmonics can be accurately described by appropriate additions to the wave equations, which are easily derived from the local dielectric tensor, and do not alter the structure of the wave equations of TORIC . Most importantly, the additional terms do not contain derivatives of the fields of order higher than second, and thus do not introduce unphysical eigenmodes which would "pollute" the numerical solution. In this section we present in some details the derivation of this model. Its implementation in TORIC extends to general distribution functions a previous version restricted to the Maxwellian case [5]

As a preliminary, let us consider harmonic IC damping in the limit of a uniform infinite plasma. The contributions of an ion species $s$ experiencing resonance at the $(p-1)$ th IC harmonic $\omega=p \Omega_{c s}$ to the hot-plasma dielectric tensor is

$$
\Delta_{s}^{p}\left[\epsilon_{i j}\right]=-\frac{\omega_{p s}^{2}}{\omega^{2}}\left(-x_{0}^{s} \tilde{Z}_{i j}\left(\xi_{\perp}^{s}, x_{p}^{s}\right)\right) \quad \xi_{\perp}^{s}=\frac{k_{\perp} v_{t h s}}{\Omega_{c s}} \quad x_{p}^{s}=\frac{\omega-p \Omega_{c s}}{k_{\|} v_{t h s}}
$$

(in the following the species superscript $s$ will be occasionally omitted). Here $\omega_{p s}$ and $\Omega_{c s}$ are the plasma and cyclotron frequency, and $v_{t h s}$ an average velocity (the thermal velocity if the species distribution function $F_{s}$ is Maxwellian). The generalized Dispersion Functions $\tilde{Z}_{i j}$ in eqn (10) have the form

$$
\tilde{Z}_{i j}\left(\xi_{\perp}, x_{p}\right)=\int_{-\infty}^{+\infty} \frac{\mathcal{F}_{i j}^{p}\left(\xi_{\perp}, u\right)}{u-x_{p}} \mathrm{~d} u
$$

with the familiar Landau prescription for the singularity, and, in the numerator,

$$
\begin{aligned}
& \mathcal{F}_{x x}^{p}=-2 \pi \int_{0}^{\infty} \frac{p^{2}}{\xi_{\perp}^{2} w^{2}} J_{p}^{2}\left(\xi_{\perp} w\right) w \frac{\partial F_{s}}{\partial w} \mathrm{~d} w \\
& \mathcal{F}_{x y}^{p}=-\mathcal{F}_{y x}^{p}=2 i \pi \int_{0}^{\infty} \frac{p}{\xi_{\perp} w} J_{p}\left(\xi_{\perp} w\right) J_{p}^{\prime}\left(\xi_{\perp} w\right) w \frac{\partial F_{s}}{\partial w} \mathrm{~d} w \\
& \mathcal{F}_{y y}^{p}=-2 \pi \int_{0}^{\infty} J_{p}^{\prime 2}\left(\xi_{\perp} w\right) w \frac{\partial F_{s}}{\partial w} \mathrm{~d} w
\end{aligned}
$$

Contributions to other components of the dielectric tensor are negligible. If the distribution function is Maxwellian these integrals can be performed in terms of the Plasma Dispersion Function $Z$ and of modified Bessel functions, respectively [21].

The power absorbed by species $s$ per unit volume from a wave of parallel wavenumber $k_{\|}$can be written in term of the antihermitian part of $\Delta_{s}^{p}\left[\epsilon_{i j}\right]$. Introducing 
rotating components of the electric field,

$$
P_{s}^{(p)}=-\frac{\omega}{8} \frac{\omega_{p s}^{2}}{\omega^{2}} x_{0} \int_{0}^{\infty}\left|J_{p-1}\left(\xi_{\perp} w\right) E_{+}+J_{p+1}\left(\xi_{\perp} w\right) E_{-}\right|^{2} w\left(\frac{\partial F_{s}}{\partial w}\right)_{u=x_{p}} \mathrm{~d} w
$$

It is worth stressing that $P_{s}^{(p)}$ is always positive as long as the distribution functions are isotropic and decrease monotonically with energy.

In TORIC the wave equation are solved by expanding the RF field in toroidal and poloidal Fourier modes [22]

$$
\vec{E}=\sum_{m, n} \vec{E}(m, n) e^{i(m \vartheta+n \varphi)}
$$

A similar expansion holds for the hf current of each charged particle species, which, in addition, is expanded in cyclotron harmonics,

$$
\overrightarrow{J_{s}}=\sum_{m, n} \sum_{p} \vec{J}_{s}^{(p)}(m, n) e^{i(m \vartheta+n \varphi)}
$$

(since in axisymmetric configuration toroidal modes propagate independently from each other, in the following we will omit to indicate the toroidal wavenumber $n$ unless needed for clarity). In principle, absorption at IC harmonics $p \geq 3$ is described by RF currents $\vec{J}_{s}^{(p)}$ which are integral functionals of the wave electric field $\vec{E}$. Our goal is to replace these integral functionals by differential expressions containing derivatives of degree not higher than second, which should satisfy the following requirements:

- $\vec{J}_{s}^{(p)}$ must be a vector;

- the local dispersion relation and the corresponding power balance must be recovered in the uniform plasma limit;

- only the Fast Wave must be affected by the additional damping.

If these conditions are satisfied, $\vec{J}_{s}^{(p)}$ will be a good approximations as long as the FW maintains its identity in the vicinity of high-order IC resonances, as is indeed always the case.

Following a method parallel to the derivation of the Finite Larmor Radius wave equations in $[23,24]$ from Vlasov equation in non-uniform plasmas, one realizes that there is only one way of satisfying these requirements, namely to write $\vec{J}_{s}^{(p)}$ for each toroidal Fourier mode in the following form

$$
\begin{array}{r}
\frac{4 \pi i}{\omega} \vec{J}_{s}^{(p)}(m)=-\frac{c^{2}}{\omega^{2}} \sum_{\bar{m}}\left\{\left(\hat{S}_{s}^{(p)}(\bar{m}) E_{\psi}(\bar{m})-i \hat{D}_{s}^{(p)}(\bar{m}) E_{\eta}(\bar{m})\right) \vec{u}_{\psi}\right. \\
+\left(i \hat{D}_{s}^{(p)}(\bar{m}) E_{\psi}(\bar{m})+\hat{S}_{s}^{(p)}(\bar{m}) E_{\eta}(\bar{m})\right) \vec{u}_{\eta} \\
\left.-2 \vec{\nabla}_{\perp} \times\left(\tau_{s}^{(p)}(\bar{m}) \vec{\nabla}_{\perp} \times \vec{E}_{\perp}(\bar{m})\right)\right\} e^{i \bar{m} \vartheta}
\end{array}
$$


where

$$
\begin{gathered}
S_{s}^{(p)}(\bar{m})=\Delta_{s}^{p}\left[\epsilon_{x x}\left(\omega, \vec{k}^{\bar{m}, n}\right)\right] \\
\tau_{s}^{(p)}(\bar{m})=\frac{\omega^{2}}{c^{2} k_{\perp}^{2}} \Delta_{s}^{p}\left[\epsilon_{y y}\left(\omega, \vec{k}^{\bar{m}, n}\right)-\epsilon_{x x}\left(\omega, \vec{k}^{\bar{m}, n}\right)\right]
\end{gathered}
$$

Here $\Delta_{s}^{p}\left[\epsilon_{i j}\right]$ denote the contributions of ion species $s$ to the local hot-plasma dielectric tensor $\underline{\underline{\epsilon}}$ introduced in the previous section; the subscripts $x, y, z$ indicate that $\epsilon_{i j}$ is written in the local "Stix frame", i.e. with $z$ axis along the static magnetic field $\left(\vec{u}_{\zeta}=\vec{u}_{z}=\vec{B}_{0} / B_{0}\right)$ and $x$-axis in the direction of $\vec{k}_{\perp}$; it is not necessary, however, to know this direction in the present context. In the argument, $k_{\perp}=k_{\perp \mathrm{FW}}$ is intended to be the solution of the local dispersion relation for the FW. We recall that in toroidal geometry $k_{\perp \mathrm{FW}}$, and more generally the elements of the local dielectric tensor depend on the poloidal number $m$ because the contribution to $k_{\|}=\vec{k} \cdot \overrightarrow{B_{0}}$ is proportional to $m$ (cfr. eq. (21) below).

By comparison with the homogeneous plasma case we immediately obtain

$$
\left(\begin{array}{c}
\hat{L}_{s}^{(p)} \\
\hat{R}_{s}^{(p)} \\
\left(c^{2} k_{\perp}^{2} / \omega^{2}\right) \tau_{s}^{(p)}
\end{array}\right)=\frac{\omega_{p s}^{2}}{\omega^{2}} x_{0}\left\{\int_{0}^{\infty}\left(\begin{array}{c}
\mathcal{F}_{+}^{(p)}\left(\xi_{\perp}, u\right) \\
\mathcal{F}_{-}^{(p)}\left(\xi_{\perp}, u\right) \\
\mathcal{F}_{\tau}^{(p)}\left(\xi_{\perp}, u\right)
\end{array}\right) \frac{\mathrm{d} u}{u-x_{p}}+i \pi\left(\begin{array}{c}
\mathcal{F}_{+}^{(p)}\left(\xi_{\perp}, x_{p}\right) \\
\mathcal{F}_{-}^{(p)}\left(\xi_{\perp}, x_{p}\right) \\
\mathcal{F}_{\tau}^{(p)}\left(\xi_{\perp}, x_{p}\right)
\end{array}\right)\right\}
$$

with $\hat{R}=\hat{S}+\hat{D}, \hat{L}=\hat{S}-\hat{D}$, and

$$
\begin{aligned}
& \mathcal{F}_{ \pm}^{(p)}\left(\xi_{\perp}, u\right)=-2 \pi \int_{0}^{\infty} \frac{p}{\xi_{\perp} w} J_{p}\left(\xi_{\perp} w\right) J_{p \mp 1}\left(\xi_{\perp} w\right) w \frac{\partial F_{s}}{\partial w} \mathrm{~d} w \\
& \mathcal{F}_{\tau}^{(p)}\left(\xi_{\perp}, u\right)=-2 \pi \int_{0}^{\infty} J_{p-1}\left(\xi_{\perp} w\right) J_{p+1}\left(\xi_{\perp} w\right) w \frac{\partial F_{s}}{\partial w} \mathrm{~d} w
\end{aligned}
$$

Here the arguments are defined as in the uniform limit, except for the fact that they depend on both the toroidal and poloidal wavenumbers:

$$
\xi_{\perp}=\xi_{\perp}^{m, n}=\frac{k_{\perp}^{m, n} v_{t h s}}{\Omega_{c s}} \quad x_{p}=x_{p}^{m, n}=\frac{\omega-p \Omega_{c s}}{k_{\|}^{m, n} v_{t h s}}
$$

with

$$
k_{\|}^{m, n}=\frac{m}{N_{\vartheta}} \sin \Theta+\frac{n}{R} \cos \Theta \quad \tan \Theta=\frac{B_{\text {pol }}}{B_{\text {tor }}}
$$

$\left(N_{\vartheta}^{2}\right.$ is the element of the metrics diagonal in the $\vartheta$ index, which reduces to the minor radius squared in the case of circular concentric magnetic surfaces).

The expression for the density of power absorption following from eqn (21), namely

$$
\begin{array}{r}
P_{s}^{(p)}(n)=\frac{\omega}{8 \pi} \sum_{m, \bar{m}} \iint R \mathcal{J}_{p} \operatorname{Im}\left\{\left[E_{+}^{*}(m) \hat{L}_{s}^{(p)}(\bar{m}) E_{+}(\bar{m})+E_{-}^{*}(m) \hat{R}_{s}^{(p)}(\bar{m}) E_{-}(\bar{m})\right.\right. \\
\left.\left.+2 \frac{c^{2}}{\omega^{2}}\left(\vec{\nabla}_{\perp} \times \vec{E}_{\perp}^{*}(m)\right) \tau_{s}^{(p)}(\bar{m})\left(\vec{\nabla}_{\perp} \times \vec{E}_{\perp}(\bar{m})\right)\right] e^{i(m-\bar{m}) \vartheta} \mathrm{d} \psi \mathrm{d} \vartheta\right\}
\end{array}
$$



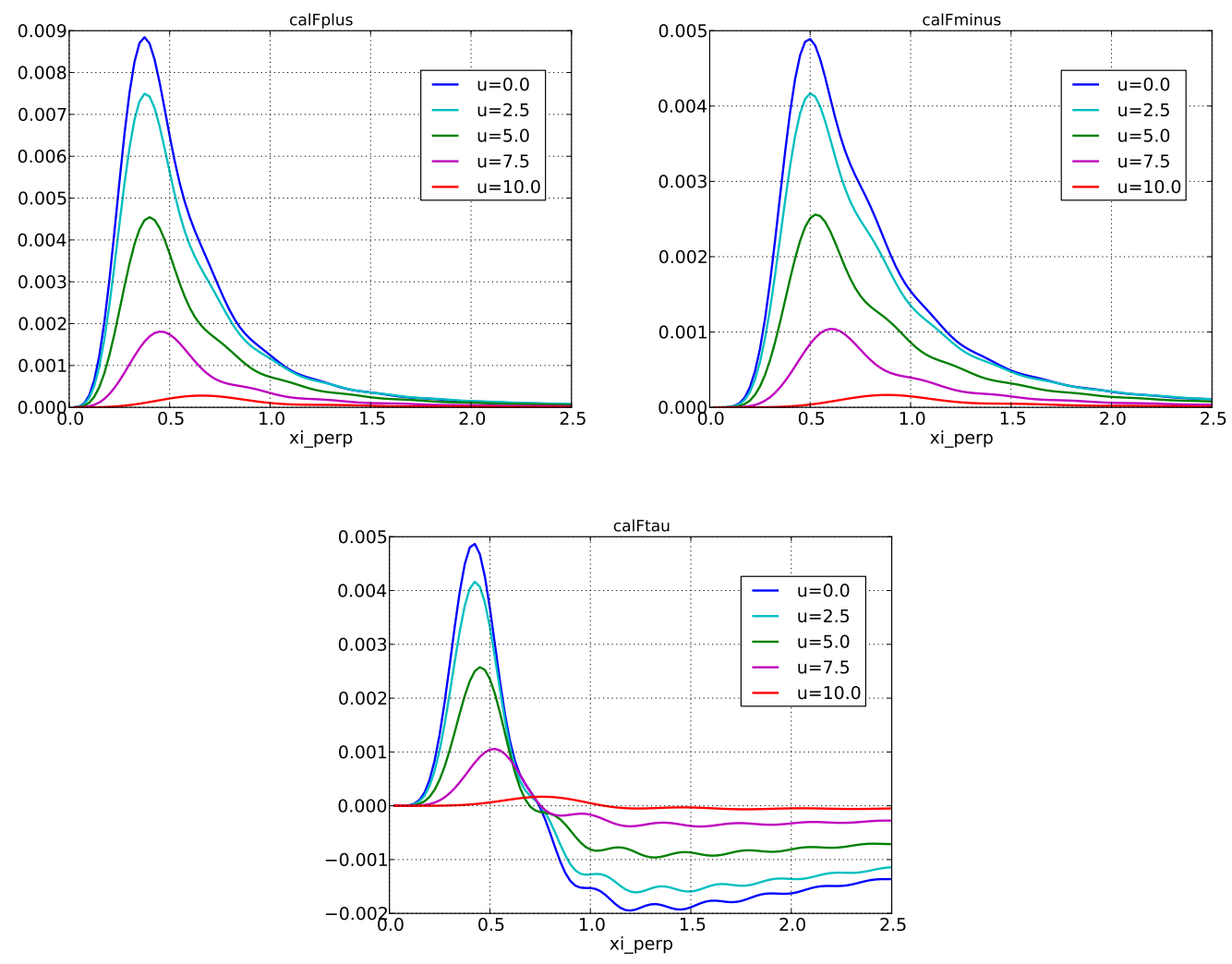

Figure 6. The functions $\mathcal{F}_{+}^{(p)}\left(\xi_{\perp}, u\right), \mathcal{F}_{-}^{(p)}\left(\xi_{\perp}, u\right)$, and $\mathcal{F}_{\tau}^{(p)}\left(\xi_{\perp}, u\right)$, for $p=3$.

is easily seen to reduce to eqn (15) in the uniform plasma limit, as required.

To apply these results to thermonuclear alpha particles, we must distinguish between the thermal and suprathermal populations. The contribution of the ${ }^{4} \mathrm{He}$ ashes can immediately be written in terms of the corresponding contributions to the appropriate elements of hot-plasma dielectric tensor of a Maxwellian plasma. We note that even at the temperature of an ignited plasma the thermal speed of thermalized $\alpha$-particles satisfies the inequality

$$
\lambda_{\alpha}=\frac{k_{\perp \mathrm{FW}}^{2} v_{t h \alpha}^{2}}{2 \Omega_{c \alpha}^{2}} \ll p
$$

by a comfortable margin; in TORIC, nevertheless, the full modified Bessel functions are taken into account in $\hat{L}_{\alpha}^{(p)}$ and $\hat{R}_{\alpha}^{(p)}$. The term $\tau_{\alpha}^{(p)}$ (a large Larmor radius correction to $\epsilon_{y y}$ without correspondence in $\epsilon_{x x}$, akin to the term describing Magnetic Pumping by the electrons, but without Cerenkov resonance) is a factor $v_{t h \alpha}^{2} / c^{2}$ smaller, and has been omitted. All other ions, except the energetic $\alpha$ population, are treated in the same way.

By contrast, the Larmor radius of a just born alpha particle can be a substantial fraction of the local FW wavelength. Slowing-down $\alpha$-particles, on the other hand, are so diluted that their contribution to the real part of the local dispersion relation is negligible (this statement has been accurately confirmed by tests using the equivalent Maxwellian, cfr. section 4.4). Accordingly, we will keep only the imaginary part of the 
quantities $\mathcal{F}^{(p)}$, eqn (24), but, of course, with the full Bessel functions taken into account in the integrals over the perpendicular velocity. These integrals take a somewhat simpler form if $v$ is used as independent variable, with $w=\left(v^{2}-u^{2}\right)^{1 / 2}$ regarded as function of $u$ and $w$ :

$$
\begin{aligned}
& \mathcal{F}_{ \pm}^{(p)}\left(\xi_{\perp}, u\right)=6 \pi K_{\mathrm{tn}} \frac{p}{\xi_{\perp}} \int_{u}^{v_{\mathrm{b}}} J_{p}\left(\xi_{\perp} w\right) J_{p \mp 1}\left(\xi_{\perp} w\right) \frac{v^{2}}{\left(v^{3}+v_{\mathrm{crit}}^{3}\right)^{2}} \mathrm{~d} v \\
& \mathcal{F}_{\tau}^{(p)}\left(\xi_{\perp}, u\right)=6 \pi K_{\mathrm{tn}} \int_{u}^{v_{\mathrm{b}}} J_{p-1}\left(\xi_{\perp} w\right) J_{p+1}\left(\xi_{\perp} w\right) \frac{w v^{2}}{\left(v^{3}+v_{\text {crit }}^{3}\right)^{2}} \mathrm{~d} v
\end{aligned}
$$

where $K_{\mathrm{tn}}$ is the normalization constant of the slowing-down function. Figures 6 show these functions for $p=3$. As functions of $u$, they extend to very large velocities, namely the birth velocity of the alpha particles. This means that the Doppler broadened absorption lines of slowing-down $\alpha$-particles are about 10 times wider than those of thermal ions. This makes slowing-down $\alpha$-particles a very efficient competitor for absorption at low IC harmonics, in spite of their very small number density.

\section{Exploring the possibility of IC range of frequency Current Drive in DEMO}

For a first orientation, we have performed a frequency scan from 20 to $300 \mathrm{MHz}$, keeping constant the key parameter which determines both the intensity of electron absorption and the efficiency of $\mathrm{CD}$, namely the ratio $\left\langle x_{e}\right\rangle=\omega /\left\langle k_{\|}\right\rangle v_{\text {the }}$ between the parallel phase velocity of the average poloidal mode of the solution $\left(m=0,\left\langle k_{\|}\right\rangle=n_{\varphi} / R\right)$, and the electron thermal speed. For each frequency we have run TORIC for three toroidal wavenumbers $n_{\varphi}$ in the ratios 1:2:3 (fig. 7; the criteria which have guided the choice of the $n_{\varphi}$ values will be mentioned shortly). Keeping $x_{e}$ constant, of course, means a different antenna periodicity at each frequency. It is also important to be aware that due to poloidal coupling, $n_{\varphi} / R$ is only the average parallel wavenumber: the actual spectrum of $k_{\|}$, hence of $x_{e}$, is always relatively broad. A convergent run of TORIC for DEMO requires at least 64 poloidal Fourier modes, $-31 \leq m \leq+31$; the corresponding ranges of $x_{e}$ along the equatorial plane for the smallest and largest value of $n_{\varphi}$ used in the scan are shown for a frequency of $50 \mathrm{MHz}$ in fig. 8. To scale these figures to other frequencies it is sufficient to recall that if the number of poloidal Fourier components is kept constant, the range of $k_{\|}$narrows inversely to the frequency.

It is apparent from fig 8 that even the lowest value of $n_{\varphi}$ selected for this scan corresponds to an average parallel phase velocity in the plasma core of the order of the electron thermal speed, hence just fast enough to avoid reduction of CD efficiency by toroidal trapping of the resonant electrons. It turns out, however, that damping of this and lower toroidal modes is dominated by IC harmonic absorption by thermonuclear alpha particles and Deuterium (in the lower part of the frequency range also by Tritium). Above $25 \mathrm{MHz}$, a few windows in which electron damping dominates can be found only 

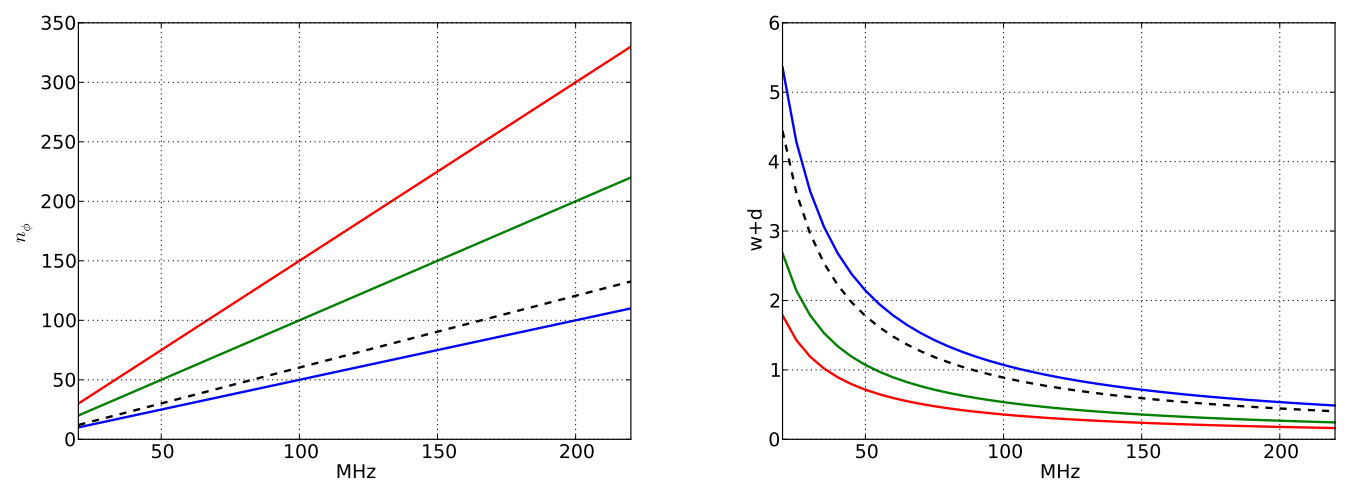

Figure 7. Left: the three values of $n_{\varphi}$ used in the frequency scan. Right: the corresponding antenna periodicity $w+d(\mathrm{~m})$. The dashed black lines correspond to the condition $x_{e}=1$ on the magnetic axis.
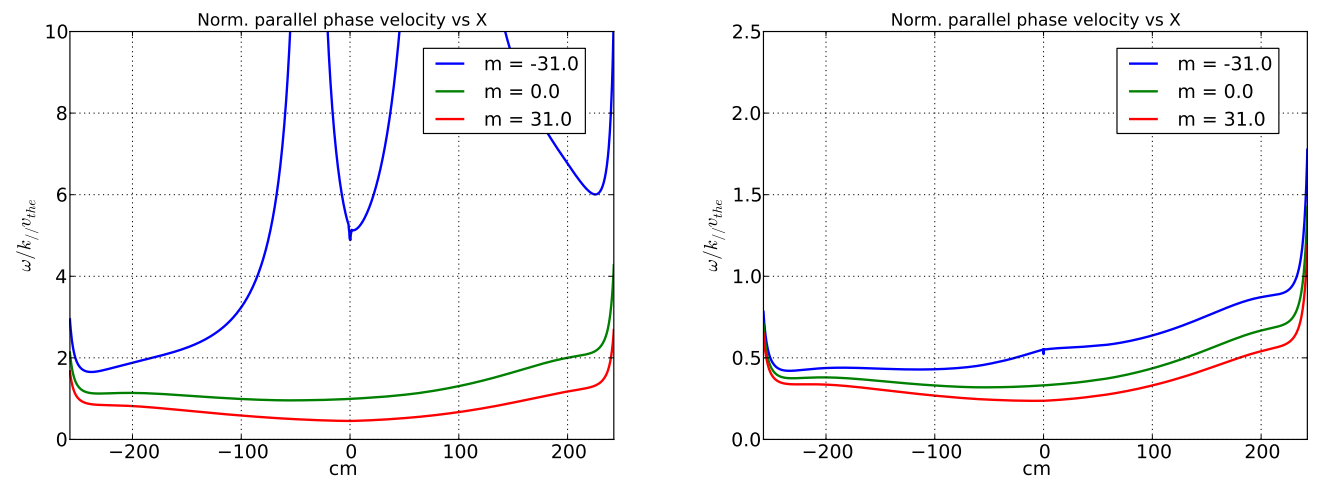

Figure 8. The range of $x_{e}=\omega / k_{\|} v_{\text {the }}$ along the equatorial plane, $f=50 \mathrm{MHz}$, left $n_{\varphi}=25$, right $n_{\varphi}=75$.

for values of $n_{\varphi}$ two times larger or more. This explains our choice of toroidal modes for the frequency exploration. It can be anticipated, on the other hand, that in this situation optimization of the driven current will depend on a rather delicate balance between the need to minimize absorption by ions, which would require toroidal wavenumbers as large as possible, and minimization of toroidal trapping of the resonant electrons, which would require parallel phase velocities as fast as possible.

The fraction of power absorbed by each species and the driven current (kA per MW coupled) are plotted as a function of frequency for the three values of $n_{\varphi}$ in figures 9 . In these figures are also shown the results obtained when the distribution function of the thermonuclear $\alpha$-particles is simulated by the equivalent Maxwellian; they will be briefly commented in the last subsection. A more complete overview of the accessible parameter space is offered by contour plots of $P_{\alpha}\left(f, n_{\varphi}\right) / P_{\text {tot }}, P_{e}\left(f, n_{\varphi}\right) / P_{\text {tot }}$, and of the driven current (kA per MW coupled) in figs 10-11 (note that the ordinate in the contour plots is $n_{\varphi} /\left(k_{0} R_{0}\right), k_{0}=\omega / c$, i.e. the parallel wavenumber on axis, so that horizontal lines are lines of constant $\left.\omega / k_{\|} v_{\text {the }}\right)$. 

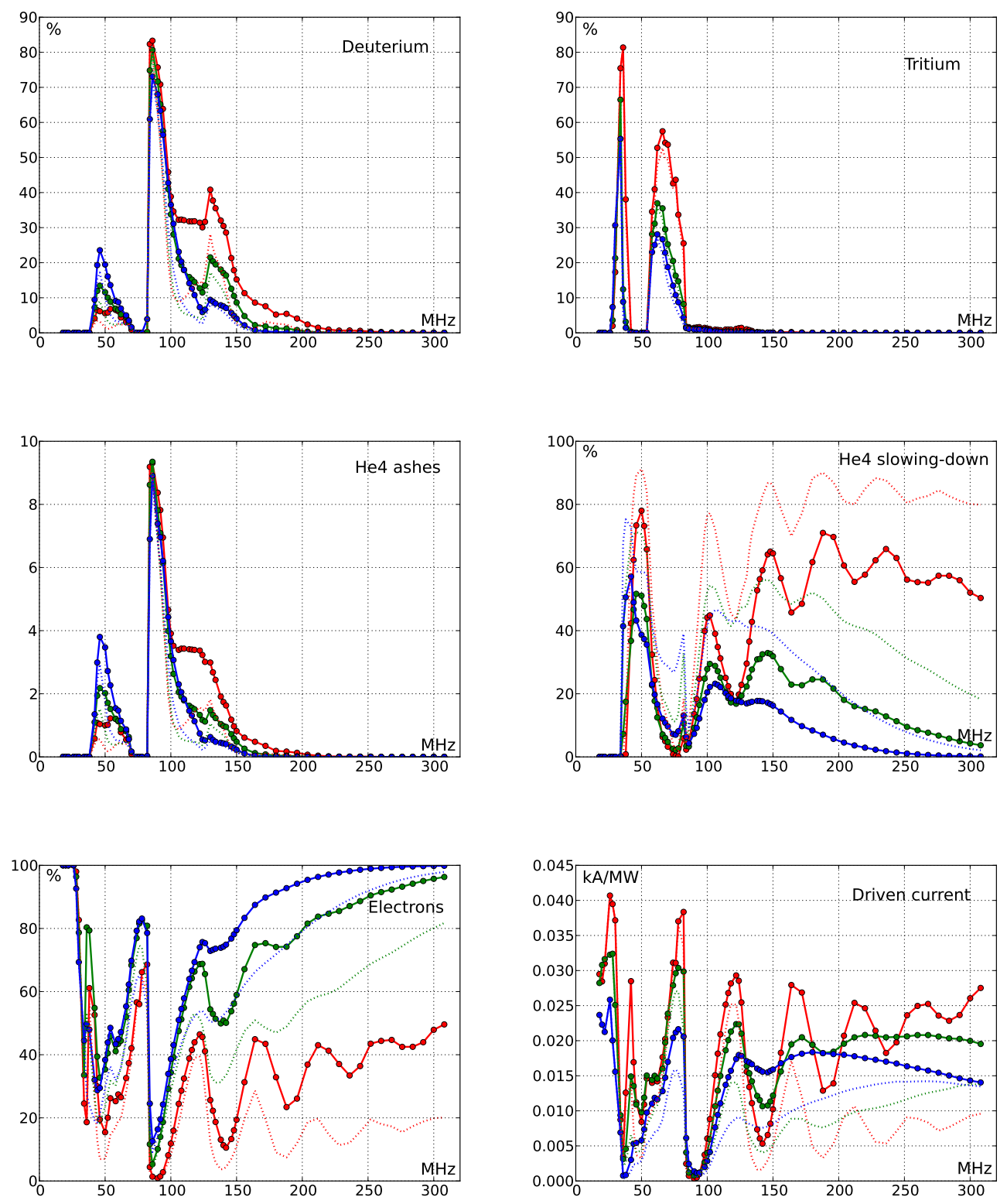

Figure 9. Fraction of power absorbed by the each species and RF driven current (kA per MW coupled), as function of frequency. In each plot the red curve is for the smallest, the green for the intermediate, and the blue for the largest $n_{\varphi}$. The dotted curves are obtained with the equivalent Maxwellian.

As the frequency increases, the entrance of successive IC harmonics in the plasma causes a steep increase of the absorption by the corresponding ion species, followed by a slower decline as the resonance in question moves farther away from the antenna. The latter is due to increasing depletion of the incoming power flux by electrons absorption in the intervening plasma, and by the entrance from the low side of IC resonances of other ion species. This kind of screening is particularly important for Tritium, which can be heated efficiently only at the fundamental and the first harmonic. Deuterium 

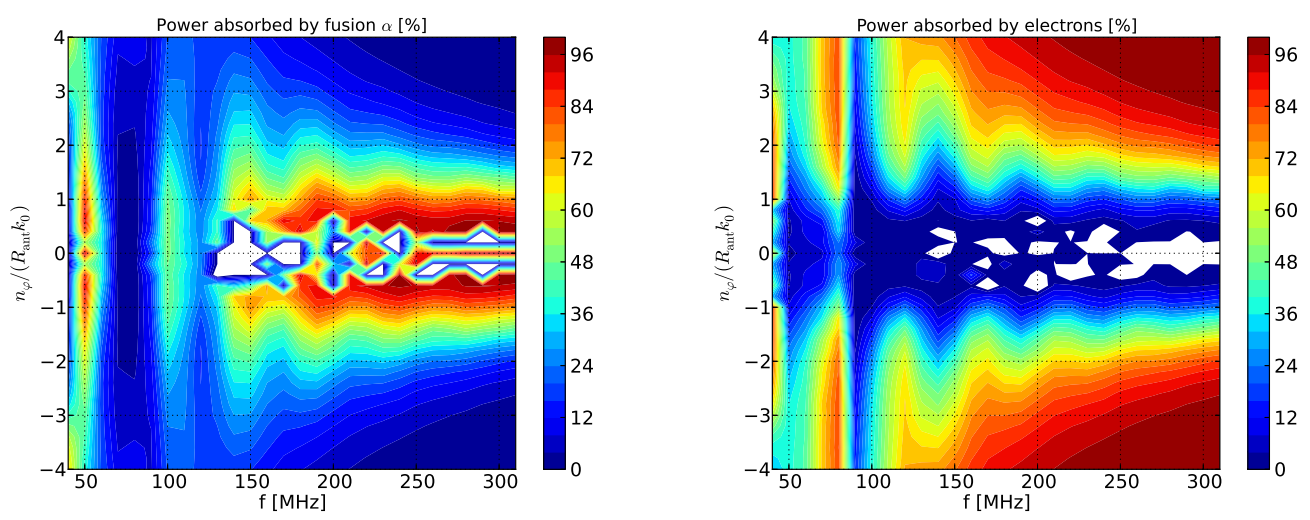

Figure 10. Fraction of power absorbed by energetic $\alpha$-particles and by the electrons as function of frequency and toroidal wave number. In the white areas absorption is poor, and the mesh used ( 801 radial points, 128 poloidal points, and poloidal modes in the range $-31 \leq m \leq+31$ ) was not sufficient to obtain a well-convergent solution.

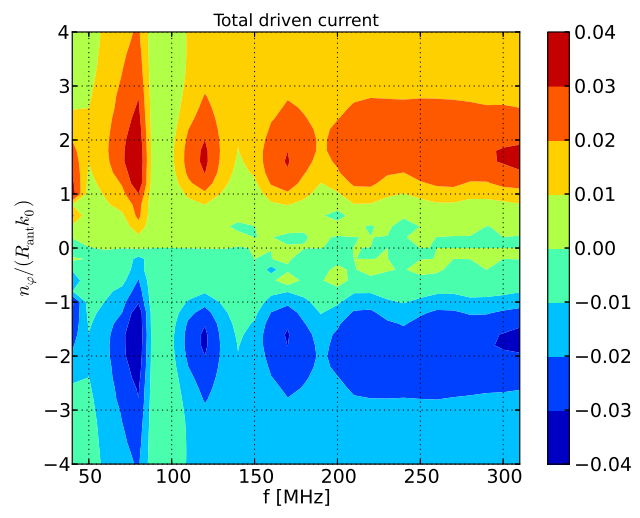

Figure 11. Driven current (kA/MW coupled) as function of frequency and toroidal wave number.

absorbs a significant fraction of power also at $\omega=3 \Omega_{c D}$; absorption at higher harmonics decreases rapidly. The frequency behaviour of absorption by thermalized $\alpha$-particles is similar, but this population absorbs only a small fraction of the total power because of its low concentration. This does not hold for energetic slowing down $\alpha$-particles, in spite of the fact that they are still much more diluted: even at the 5th harmonic they can take half or more of the power radiated in modes with low to moderate toroidal wavenumbers. As anticipated, we find that such dominant absorption by energetic $\alpha$ particles can be avoided only by launching toroidal modes whose average phase velocity is well below the electron thermal speed in the plasma core.

In fig. 9 are also shown the results for the frequency scan with three toroidal modes obtained when the distribution function of the $\alpha$-particles is simulated with the equivalent Maxwellian. As anticipated, this model reproduces the trends, but grossly overestimates absorption by the energetic $\alpha$-population, particularly at high IC harmonics. This can be understood by noting (cfr. fig. 3) that the equivalent Maxwellian 
has more ions at low energies and less at high energies, hence on average a larger gradient in velocity. We conclude that the equivalent Maxwellian approximation should not be used for quantitative predictions. It has been brought to our attention during the revision of the manuscript that a comparison of IC absorption by $\alpha$-particles evaluated with the equivalent Maxwellian and with the slowing-down distribution performed by R. Koch [25] reaches conclusions partly in disagreement with the results of fig. 9, and suggests that a different definition of the equivalent Maxwellian might give better agreement. This suggestion, however, was based on the integration of $\operatorname{Im}\left(\epsilon_{x x}\right)$ over the Doppler IC absorption lines for a single value of $k_{\perp}$ and $k_{\|}$(the same for all harmonics considered, namely the first three), so that it is difficult to conclude about its general validity.

Since with the equivalent Maxwellian the contributions of the thermonuclear $\alpha$ particles to the coefficients of the wave equations are expressed in terms of the Plasma Dispersion Function $Z\left(x_{p}\right)$, it has been easy to check that the results obtained by including or omitting the Hermitian part of these contributions are practically identical. It is very plausible, therefore, that omission of the Hermitian contribution is fully justified also in the simulations which use the more realistic slowing-down distribution function.

Figure 11 shows that for the parameters of DEMO the CD efficiency (defined here as kA driven per MW coupled) is maximum at values of $n_{\varphi} / k_{0} R_{0} \sim \pm 2$, in the frequency ranges where competition by absorption by ions is not too strong. These maxima in the CD efficiency, however, cannot necessarily be exploited, either because they are in a domain where absorption by ions is nevertheless unacceptably large, or because they put too severe requirements on the antenna geometry. Analysis of figs. 9-11 taking these constraints into account suggests three possible frequencies windows for CD, already noted by Lerche et al. [10], namely below the fundamental IC resonance of all ion species $(f \lesssim 24 \mathrm{MHz}$ in DEMO), in a narrow range around 75 to $80 \mathrm{MHz}$ depending on the poloidal position of the antenna, and above about 180-200 MHz. We now review each of these windows in some more detail, considering also the demands on the antenna design.

\subsection{The low-frequency range}

The most obvious window for RF current drive is below the IC resonances of all ion species. In this range, however, any antenna compatible with the available access necessarily selects toroidal wavenumbers corresponding to parallel phase velocities much slower than the electron thermal velocity. Indeed, at $20 \mathrm{MHz}$ the condition $\omega / k_{\|} v_{\text {the }} \geq 1$ in the plasma core of DEMO would require $n_{\varphi} \lesssim 0.6 f_{\mathrm{MHz}} \simeq 12$. It is easily seen that no antenna compatible with the available access can select so low toroidal numbers.

As an example of what is possible in this range, the spectrum radiated a 4 element antenna of total toroidal length of $1.36 \mathrm{~m}$ (strap width $b=0.18 \mathrm{~m}$, gap between straps $d=0.20 \mathrm{~m}$ ), excited with phase difference $\Delta \phi=\pi / 2$ between adjacent straps, is shown 

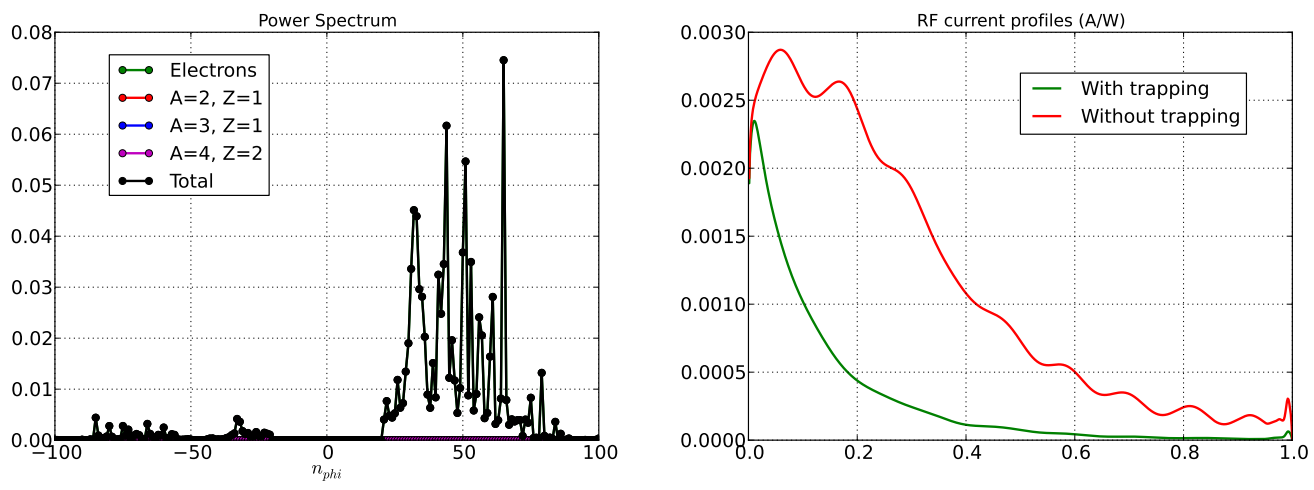

Figure 12. Low frequency range $(20 \mathrm{MHz})$. Left: the power spectrum radiated by a 4 strap antenna (strap width $b=0.18 \mathrm{~m}$, gap between straps $d=0.20 \mathrm{~m}$ ) in the $(0, \pi / 2, \pi, 3 \pi / 2)$ configuration (at this frequency, all power is absorbed by the electrons). Right: radial profile of the RF-induced current by this antenna. The toroidal spectrum has been obtained by solving Maxwell's equations in parallel for all toroidal modes in the range $-100 \leq n_{\varphi} \leq+100$.

in fig. 12 a). It is peaked at $n_{\varphi} \simeq 50$, corresponding to $\omega /\left\langle k_{\|}\right\rangle v_{\text {the }} \lesssim 0.2$ over most of the plasma. As a consequence, most resonant electrons are trapped, resulting in a prohibitive reduction of the driven current, as shown in fig. $12 \mathrm{~b}$ ), namely from the relatively large value of $86.1 \mathrm{kA} / \mathrm{MW}$ obtained omitting toroidal effects to $12.2 \mathrm{kA} / \mathrm{MW}$ when they are taken into account. This figure shows that toroidal trapping of resonant electrons, in addition to spoiling the efficiency, also drastically modify the expected driven current profile, since they get stronger away from the magnetic axis.

\subsection{The high frequency range}

In the high-frequency window the optimization of the driven current and the imperative to avoid excessive absorption by the ions put opposite demands on the launched power spectrum. Finding a workable compromise between these two requirements is made difficult by the the fact that the needed antenna dimensions become uncomfortably small as the frequency increases.

To illustrate the problems encountered, we have made a complete scan over toroidal modes at $220 \mathrm{MHz}$, covering the range $-400 \leq n_{\varphi} \leq 400$, both with equatorial launching and with the antenna located $75^{\circ}$ above the mid plane, and considered in each case a set of 16 strap antennas, all with a gap of $2 \mathrm{~cm}$ and phase difference $\pi / 2$ between adjacent elements, and varying the width of the individual conductors from 12 to $4 \mathrm{~cm}$ (note that such a multi-strap antenna cannot be built as a combline, since to obtain the appropriate relative phasing all elements must be active). The results obtained are summarized in fig. 13. That the fraction of power absorbed by the electrons saturates and even decreases for the narrowest straps, i.e. slower average parallel phase velocities, might at first seem to contradict the contour plots of fig. 10. The reason is that for such narrow straps satellite peaks at values of $n_{\varphi}$ below the nominal maximum increasingly spoil the 


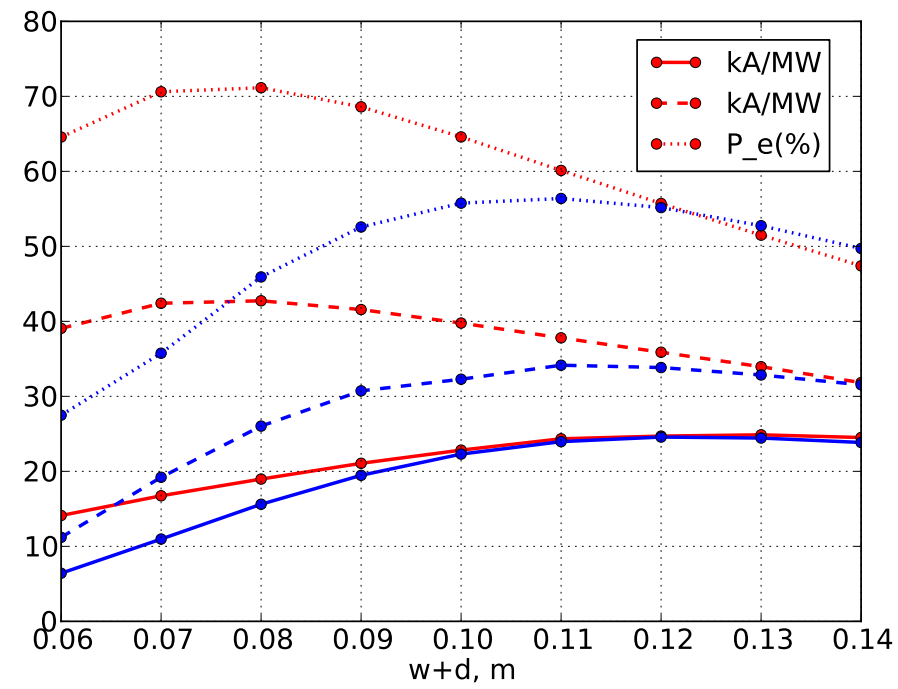

Figure 13. High frequency range $(220 \mathrm{MHz})$ : driven current (kA per $\mathrm{MW}$ coupled) versus antenna dimensions (strap width $w$, gap $d, 16$ straps with relative phase $\pi / 2$.). Blue curves: upper antenna; red curves: equatorial antenna (the dashed lines show the same estimates when toroidal trapping of resonant electrons is neglected). The dotted curves give the fraction of the total power absorbed by the electrons.
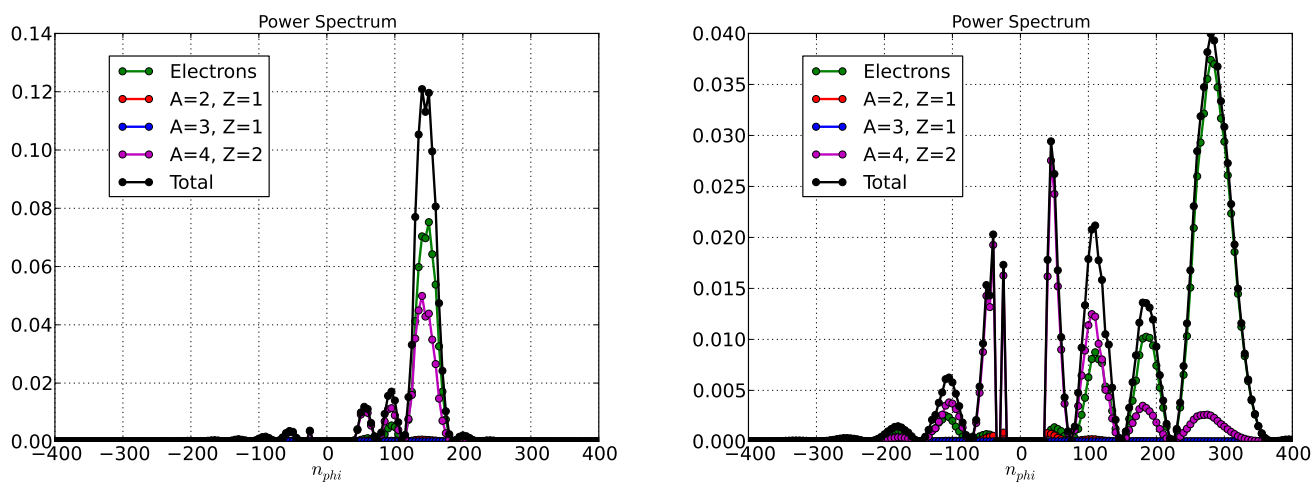

Figure 14. High frequency range $(220 \mathrm{MHz})$ : power spectra of a 16 strap launcher, $d=2 \mathrm{~cm}, \Delta \phi=\pi / 2$. Left: $w=10 \mathrm{~cm}$; right: $w=4 \mathrm{~cm}$. The toroidal spectrum has been obtained by solving Maxwell's equations in parallel for one toroidal mode every 10 in the range $-400 \leq n_{\varphi} \leq+400$. The results of a few modes around $n_{\varphi}=0$ have been skipped, because TORIC could not reach the desired accuracy due to the very low absorption per transit. 
selectivity of the structure, as can be seen by comparing the spectra with $w=10 \mathrm{~cm}$ and $w=4 \mathrm{~cm}$ in fig. 14 .

As a consequence of the difficulty of properly shaping the spectrum at these high frequencies, the maximum driven current obtained is uncomfortably low. Some unconventional idea on antenna design seems to be needed to make this range accessible for FWCD, and it will in any case be difficult to overcome the limitations due to the necessity of compromising between minimization of ion absorption on the one hand, and of toroidicity effects on the CD efficiency on the other.

\subsection{The intermediate frequency range}

The most promising window for CD in DEMO seems to be around $75 \mathrm{MHz}$ to $80 \mathrm{MHz}$, depending on the location of the antenna. In this narrow range absorption by the electrons gets competition mainly from the weak first harmonic IC resonance of Tritium, located about half a meter to the high-field side of the magnetic axis. The first harmonic of Deuterium and Helium is still outside the plasma on the low-field side, although the Doppler shift of thermonuclear $\alpha$-particles is large enough to cause a modest damping (1-2\%) by this population just below the antenna. An advantage of this window is that conventional multi-strap antennas can be used to launch a spectrum in the optimal range of parallel phase velocities. On the negative side it has to be mentioned that the window is narrow, and its position in frequency depends sensitively on the value of the static magnetic field.

As an example, using the output of a scan of toroidal modes in the range $-200 \leq n_{\varphi} \leq+200$, we have considered a set of antennas with 6 elements, excited with a phase difference of $\pi / 2$ between adjacent straps, varying the strap width $w$ from 6 to $18 \mathrm{~cm}$, with a gap $d$ each time $2 \mathrm{~cm}$ wider than the width. In fig. 15 we compare the results with the antenna in the equatorial plane at $75 \mathrm{MHz}$, and with the antenna in the upper part of the outer vessel, $75^{\circ}$ above the equatorial plane, at $80 \mathrm{MHz}$. The latter antenna clearly achieves a somewhat higher efficiency, with a shallow maximum at a $w+d=22 \mathrm{~cm}$. For comparison with other similar studies, this optimum corresponds to a value of the global efficiency parameter [1]

$$
\left\langle\gamma_{\mathrm{CD}}\right\rangle=\frac{n_{\mathrm{avg}} R_{0} I_{\mathrm{CD}}}{P_{\mathrm{hf}}} \simeq 0.23
$$

( $n_{\text {avg }}$ in units of $10^{20} \mathrm{~m}^{-3}$ ). The reason for the slight advantage of the upper antenna is not obvious. The fraction of power absorbed by the electrons is not much different between the two antennas; and the radial profile of the current driven from the equatorial plane is appreciably more peaked towards the magnetic axis (fig. 16), which should imply a less severe reduction of the efficiency by toroidicity. An explanation is suggested by the observation that the efficiency depends so weakly from the antenna periodicity (the scan of fig. 15 cover almost a factor three in $w+d$ ). This indicates that the electron absorption profile is determined by a rather subtle balance between the value of $\omega / k_{\|} v_{\text {the }}$ along the wave path, and the length of the path itself. Waves from the 


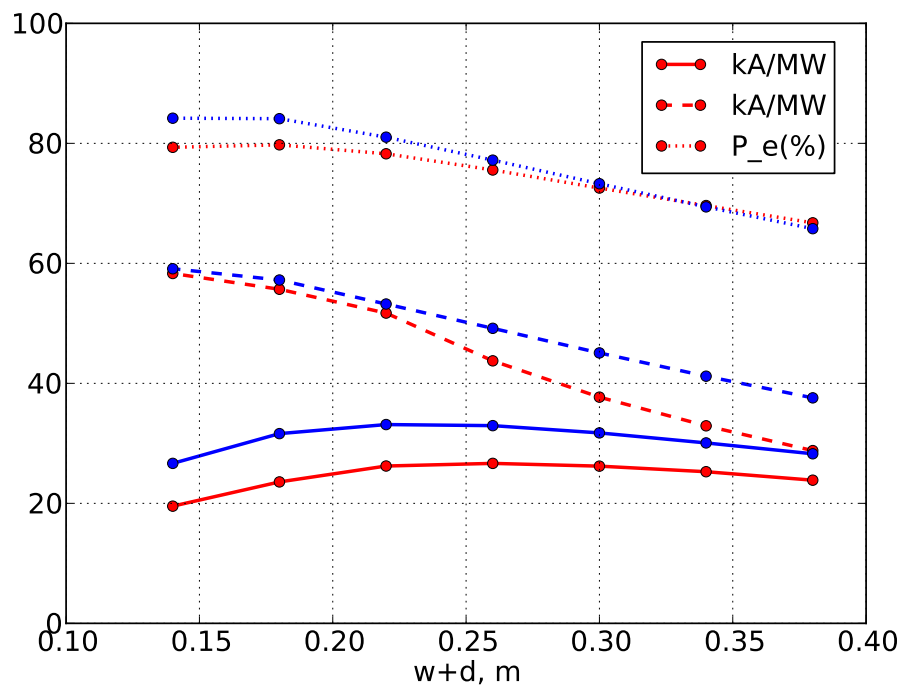

Figure 15. Intermediate frequency range: driven current (kA per MW coupled) versus antenna dimensions (strap width $w$, gap $d, 6$ straps). Blue curves; upper antenna, $80 \mathrm{MHz}$; red curves: equatorial antenna, $75 \mathrm{MHz}$ (the dashed lines show the same estimates when toroidal trapping of resonant electrons is neglected). The dotted curves give the fraction of the total power absorbed by the electrons.
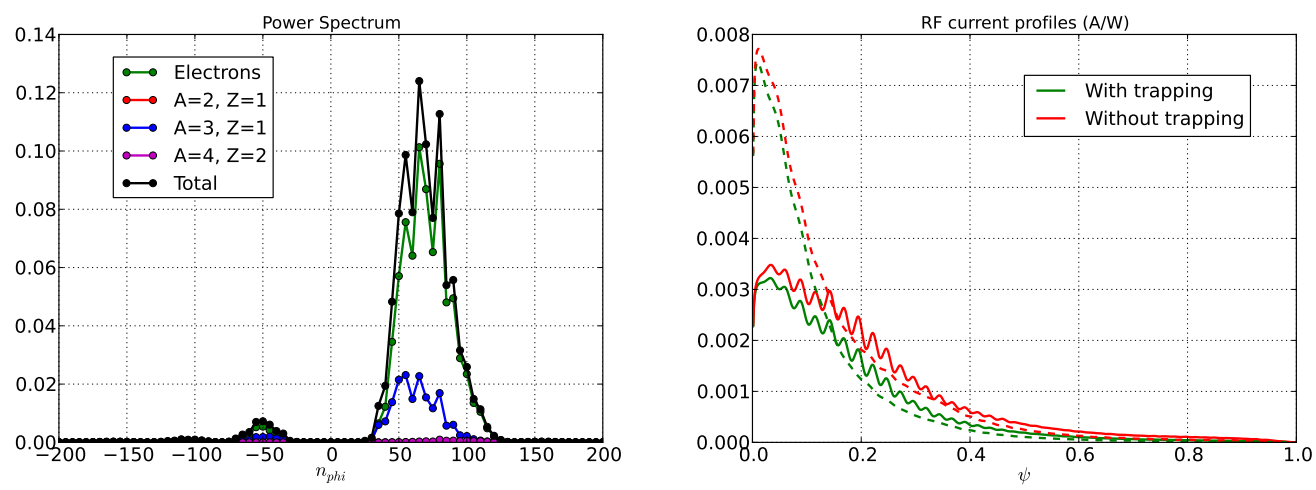

Figure 16. Intermediate frequency range ( $80 \mathrm{MHz}, 6$-strap antenna located $75^{\circ}$ above the mid plane, $w=10 \mathrm{~cm}, d=12 \mathrm{~cm}, \Delta \phi=\pi / 2)$. Left: Power spectrum. Right: radial profile of the driven current (the dashed curved are for the same antenna at $f=75 \mathrm{MHz}$, on the equatorial plane).

upper antenna traverse an appreciably longer plasma layer before reaching the center, allowing absorption of Fourier components with higher parallel phase velocity, and thus more weakly damped but more efficient in driving current, than in the case of equatorial launching. If correct, on the other hand, this explanation suggests in turn that the comparison between the two antenna locations might be rather sensitive to the electron temperature and density profiles: more simulations with different plasma parameters (and reliable predictions about the parameters of the real DEMO plasma) will be needed to reach a final conclusion on this point. 


\section{Conclusions}

Using new developments of the TORIC code, in particular an adequate modeling of FW absorption by very energetic thermonuclear $\alpha$-particles with markedly non-Maxwellian distribution function, and the parallelization allowing the solution of the wave equations for many toroidal modes simultaneously, we have explored the prospects of current drive in the ignited DEMO using Fast Waves in the Ion Cyclotron range of frequencies, keeping in mind also the constraints imposed by the physics on the antenna design. These new tools manifestly allow a much more realistic estimate of the achievable CD efficiency than previously possible.

The value of $\left\langle\gamma_{\mathrm{CD}}\right\rangle$ cited above, although appreciably lower than that achievable with Lower Hybrid waves, is still quite acceptable if an appropriate launcher can be designed. This global figure of merit, on the other hand, is not the only criterion for the choice of the CD system in ignited plasmas. Use of the compressional wave in this frequency range would have some advantages. High power generators are available, penetration to the plasma core is easy, and, at least compared with heating and current drive method at higher frequencies, even launching is somewhat simpler. On the other hand, absorption predominantly on the electrons, as required for current drive, turns out to be possible only in limited frequency windows, and for parallel phase velocity lower than the electron thermal speed. The latter constraint implies a reduction of the CD efficiency by toroidal trapping of the resonant electrons, which turns out to be prohibitive in the lowest frequency window $(f \lesssim 25 \mathrm{MHz})$, and severe in the high frequency one $(f \gtrsim 200 \mathrm{MHz})$. Only in a narrow intermediate window, which for the DEMO parameters is located at $75-80 \mathrm{MHz}$, it appears possible to achieve a satisfactory CD efficiency with a conventional multi-strap antenna, better if located in the upper part of the vessel rather than in the equatorial plane.

A similar investigation for ITER [26] is underway. First explorations indicate that frequency windows for CD in ignited ITER plasmas are somewhat wider and easier to exploit than in DEMO, except in minority heating scenarios (e.g. DT $\left({ }^{3} \mathrm{He}\right)$ plasmas) if the minority concentration remains the same after ignition. This confirms and extends to higher IC harmonics previous results [27, 28, 29].

The most important limitations of the model used in this work are the neglect of quasilinear effects on distribution function of fast $\alpha$-particles, and of radial diffusion during slowing-down in the determination of their density profile. Investigations of the sensitivity of our results to the antenna and the equilibrium parameters, for example considering a peaked rather than flat density profile in DEMO, are underway, and will

be presented elsewhere [30]. The antenna model used in TORIC is very simple, but we do not expect a more sophisticated model to appreciably change our conclusions. An improved model will be needed, on the other hand, when the time will come to optimize the antenna design. 


\section{Acknowledgments}

We wish to thank Dr. C. Angioni for enlightening discussions on the distribution function of slowing-down thermonuclear alpha particles. We are indebted to Dr. G. Tardini and Dr. E. Poli for the standard DEMO equilibrium and for discussions on the parameters of this project. We acknowledge useful discussions with Dr Y. Kazakov and his careful reading of the manuscript. A long-date collaboration with Dr. P. Bonoli and Dr. J.C. Wright for improvements to the TORIC code is also gratefully acknowledged. 
[1] Zohm H, Barbato E, Jenkins I, Kemp R, Lerche E, Poli E, Tardini G and v Eester D 2013 Assessment of h\&cd system capabilities for demo 40th EPS conference p O3.108

[2] Zohm H, Angioni C, Fable E, Federici G, Gantenbein G, Hartmann T, Lackner K, Poli E, Porte L, Sauter O, Tardini G, Ward D and Wischmeier M 2013 Nuclear Fusion 53073019

[3] Brambilla M and Bilato R 2009 Nuclear Fusion 49085004 URL http://stacks.iop.org/0029-5515/49/085004

[4] Bilato R, Brambilla M, Maj O, Horton L, Maggi C and Stober J 2011 Nuclear Fusion 51103034

[5] Brambilla M 2002 Plasma Phys. Contr. Fusion 442423

[6] Ehst D and Karney C 1991 Nucl. Fusion 311933

[7] Wright J, Phillips $\mathrm{C}$ and Bonoli $\mathrm{P} \quad 1997$ Nuclear Fusion $37 \quad 1349$ URL http://stacks.iop.org/0029-5515/37/i=10/a=I02

[8] Bilato R, Brambilla M, Pavlenko I and Meo F 2002 Nuclear Fusion 42 1085-1093

[9] Prater R, Moeller C, Pinsker R, Porkolab M, Meneghini O and Vdovin V 2014 Nuclear Fusion 54 083024 URL http://stacks.iop.org/0029-5515/54/i=8/a=083024

[10] Lerche E, Van Eester D, Messiaen A, Franke $T$ and contributors E P 2014 AIP Conference Proceedings $\mathbf{1 5 8 0} 338-341$ URL http://scitation.aip.org/content/aip/proceeding/aipcp/10.1063/1.4864557

[11] Hannan A, Hellsten T and Johnson T 1913 Nuclear Fusion 53043005

[12] Choi M, Green D, Heidbrink W W, Harvey R, Liu D, Chan V S, Berry L A, Jaeger F, Lao L L, Pinsker R I, Podesta M, Smithe D N, Park J M, Bonoli P, SciDAC R and Team S 2010 Physics of Plasmas 17056102 URL http://link.aip.org/link/?PHP/17/056102/1

[13] Angioni C, Peerers A, Perverzev G, Bottino A, Candy J, Dux R, Fable E, Hein T and Waltz R 2009 Nuclear Fusion 49055013

[14] Post D, Mikkelsen D, Hulse R, Stewart L and Weisheit J 1981 Journal of Fusion Energy 1 129-142 ISSN 0164-0313

[15] Zweben S, Budny R and et al D D 2000 Nuclear Fusion 4091

[16] Stix T 1975 Nuclear Fusion 15737

[17] Bilato R, Brambilla M and Poli E 2014 Physics of Plasmas (1994-present) 21104502 URL http://scitation.aip.org/content/aip/journal/pop/21/10/10.1063/1.4897323

[18] Brambilla M 1994 Nuclear Fusion 34 1121-43 URL http://stacks .iop.org/0029-5515/34/1121

[19] Budny R, Berry L, Bilato R, Bonoli P, Brambilla M, Dumont R, Fukuyama A, Harvey $\mathrm{R}$, Jaeger $\mathrm{E}$, Indireshkumar $\mathrm{K}$, Lerche $\mathrm{E}$, McCune $\mathrm{D}$, Phillips $\mathrm{C}$, Vdovin $\mathrm{V}$, Wright $J$ and members of the ITPA-IOS 2012 Nuclear Fusion $\mathbf{5 2} 023023$ URL http://stacks.iop.org/0029-5515/52/i=2/a=023023

[20] Kazakov Y, Eester D V, Lerche E, Wauters T and Ongena J 2014 Potential of fast wave icrf current drive in demo plasmas 40th EPS conference p 2.031

[21] Brambilla M 1998 Kinetic theory of plasma waves vol 1 (Oxford University Press)

[22] Brambilla M 1999 Plasma Physics and Controlled Fusion 41 1-34 URL http://stacks.iop.org/0741-3335/41/1

[23] Brambilla M and Ottaviani M 1985 Plasma Phys. Contr. Fusion 27919

[24] Brambilla M 1991 Plasma Phys. Contr. Fusion 331048

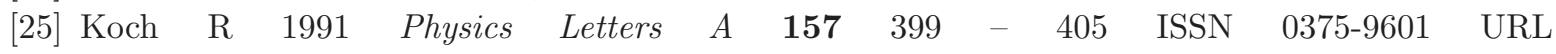
http://www.sciencedirect.com/science/article/B6TVM-46SXNDK-1G7/2/dcba9af9df2a2782c0aac0682c047538

[26] Gormezano C, Sips A, Luce T, Ide S, Becoulet A, Litaudon X, Isayama A, Hobirk J, Wade M, Oikawa T, Prater R, Zvonkov A, Lloyd B, Suzuki T, Barbato E, Bonoli P, Phillips C, Vdovin V, Joffrin E, Casper T, Ferron J, Mazon D, Moreau D, Bundy R, Kessel C, Fukuyama A, Hayashi N, Imbeaux F, Murakami M, Polevoi A and John H S 2007 Nuclear Fusion 47 S285-S336 URL http://stacks.iop.org/0029-5515/47/S285

[27] Eester D V, Louche F and Koch R 2002 Nuclear Fusion 42 310-328 URL http://stacks.iop.org/0029-5515/42/310

[28] Jaeger E F, Berry L A, D'Azevedo E F, Barrett R F, Ahern S D, Swain D W, Batchelor 
D B, Harvey R W, Myra J R, D'Ippolito D A, Phillips C K, Valeo E, Smithe D N, Bonoli P T, Wright J C and Choi M 2008 Physics of Plasmas 15072513 (pages 10) URL http://link. aip.org/link/?PHP/15/072513/1

[29] Dumont R and Zarzoso D $2013 \quad$ Nuclear Fusion 53013002 URL http://stacks.iop.org/0029-5515/53/i=1/a=013002

[30] Bilato R, Brambilla M and Fable E 2014 accepted in Journal of Physics: Conference Series 